\title{
Study on the Transonic Flows around a Thin Wing with an Aileron
}

\author{
By Masato TAmayama, ${ }^{1)}$ Hamid Reza KheIRAndish ${ }^{2)}$ and Jiro NAKAmichi ${ }^{1)}$ \\ ${ }^{1)}$ National Aerospace Laboratory of Japan, Mitaka, Japan \\ ${ }^{2)}$ Research Center of Computational Mechanics, Inc., Tokyo, Japan
}

(Received November 5th, 2002)

\begin{abstract}
The experimental airplanes of Supersonic Transportation, SST, are being developed in Japan. The wings are thin to augment cruise aerodynamic performance. If these wings are equipped with ailerons, the shock wave motions on the ailerons might cause aileron buzz not accompanied by heavy separation of the boundary layers. On the other hand, unsteady aerodynamics research regarding thin wings has not been extensive. In order to precisely investigate the shock wave motions on thin wings, two-dimensional CFD simulations were conducted in our study. In the simulations, Navier-Stokes equations are solved around the NACA0003 airfoil with an oscillating aileron. The results show that the imaginary component of the unsteady aileron hinge moment, abbreviated "AHMI," has the maximum and positive value when the shock wave oscillates around the mid-chord on the aileron. The aileron length does not strongly affect the shock wave motions on the aileron if the scale of the shock wave motions is normalized with the aileron length. But a longer aileron shows larger AHMI than a shorter one. The experimental results on SST arrow wing are compared with simulated results, too. In order to compare the results acquired from the models having different space dimensions, the local Mach number component normal to the aileron hinge line is calculated as a common parameter. The comparison shows that, for both results, AHMI becomes positive when the shock wave oscillates around the aileron mid-chord. In addition, as the shock wave oscillates more rearward on the aileron, AHMI increases.
\end{abstract}

Key Words: Transonic Flow, Aileron Buzz, Thin Wing, Unsteady Aerodynamics

\section{Nomenclature}

$$
\begin{aligned}
& b: \text { semi-chord length } \\
& c \text { : chord length } \\
& C_{M \text { hinge_unst }} \text { : coefficient of unsteady aileron hinge moment } \\
& C_{p}: \text { steady pressure coefficient } \\
& C_{p_{-} \text {unst }} \text { : unsteady pressure coefficient } \\
& C_{p}^{\prime} \text { : real component of } C_{p-\text { unst }} \\
& C_{p}: \text { : imaginary component of } C_{p \_ \text {unst }} \\
& C_{p_{-} \text {amp }}: \text { amplitude of } C_{p \_ \text {unst }} \\
& C_{p \text { _unst L }} \text { : unsteady pressure coefficient on airfoil lower } \\
& C_{p_{\_} \text {unst_U }}: \text { unsteady pressure coefficient on airfoil upper }
\end{aligned}
$$

(C) 2003 The Japan Society for Aeronautical and Space Sciences leading edge

$x_{\mathrm{h}}$ : chord-wise location of aileron hinge

$\alpha$ : angle of attack

$\beta$ : angle of the local flow against the $x$ axis

$\delta$ : aileron deflection angle

$\delta_{0}:$ mean deflection angle of aileron oscillation

$\delta_{\text {amp }}:$ amplitude of aileron oscillation

$\gamma:$ angle of aileron leading edge against the span wise axis

$\mu$ : viscosity

$\theta$ : phase lag of $C_{p \text {-unst }}$ from aileron motion

$\rho:$ free-stream density

\section{Introduction}

Next-generation SST is now gathering a lot of attention in Japan, and the flight models are going to be launched in Woomera, Australia. The no-propulsive flight model will be launched in 2002. Prior to manufacturing this flight model, aeroelasticity simulations were conducted. The results show that no unstable aeroelasticity phenomena appear in the flight envelope, but that aileron buzz might occur near the flight envelope. ${ }^{1)}$ This phenomenon is mainly caused by characteristics related to the cross-section and planform of the SST wing. The main surface of the SST model is characterized by a thin and low-aspect-ratio wing. When the angle of attack is small, the pressure gradient on a thin wing is usually small. At this condition, the boundary layer on the airfoil weakly interacts with a shock wave and hardly separates from the wing. The influence of aileron buzz depends 
on whether or not the flow is attached to the wing. Lambourne classified aileron buzz into three types: Regions A, $\mathrm{B}$, and $\mathrm{C}^{2}{ }^{2}$ Region A buzz occurs when a shock wave exists upstream of the aileron hinge with boundary layer separation. Region B buzz occurs with a shock wave oscillating on the aileron. Region $\mathrm{C}$ buzz occurs when a shock wave reaches the trailing edge of the aileron. Flow separation does not necessarily cause Region B and C buzz. Region A buzz is observed in the transonic flows on relatively thick wings because those flows are often accompanied by strong shock waves, which cause a shock-boundary-layer interaction. Thick wings are usually used for transonic airplanes, and a lot of attention has been given to Region A buzz. On the other hand, Region B buzz is most probable for SST configurations because, as presented in the experimental study ${ }^{3-5}$ ) on a SST arrow wing, a shock wave appears at first on the aileron as Mach number increases. Region B buzz grows its oscillating amplitude rapidly and drops easily into a hard flutter. ${ }^{6)}$ Alleviation of Region B buzz is crucial in SST development. Not only stiffening the aileron hinge, but also applying mass balancing is hardly adaptable to thin wings because of little spatial room around the aileron structure. Therefore, other techniques are needed to prevent Region B buzz. The essential phenomenon of Region B buzz is the shock wave motion on the aileron. In order to develop a high-performance lifting surface free from Region B buzz, which will be expected for next-generation SST, precise studies are needed on the relationship between the shock wave motion on the aileron and the aerodynamic forces. Taking a contrary position on this necessity, little research has been done on the unsteady flows relating Region B buzz. There are no papers that provide the data of shock wave oscillations on thin wings.

The main objective of this paper is to investigate the influences of shock wave motion upon aileron hinge moment. This study is based on two-dimensional CFD simulations about an airfoil with an aileron in forced oscillations, ${ }^{7)}$ in which Navier-Stokes equations are solved. The airfoil section used in this study is NACA0003 with an aileron, which is $0.25 c$ in length and $0.094 c$ as well. Several cases were studied varying the Mach number and the mean aileron deflection angle chosen as the parameters. The imaginary component of the aileron hinge moment (AHMI) becomes almost positive when the shock wave oscillates on the aileron, meaning that the surrounding flow provides the aileron with energy. If the shock wave oscillates around the mid-chord of the aileron, AHMI shows the maximum value. If the mean shock wave location and its oscillating amplitude are normalized with the aileron length, the relation between them varies only slightly between different aileron lengths. Three-dimensional experimental results are compared with simulated results as well. For both the simulated and the experimental results, AHMI becomes positive when the shock wave oscillates around the aileron mid-chord, and AHMI increases as the shock wave oscillates more rearward on the aileron.

\section{Method of Simulation}

In this study, the CFD code developed by Kheirandish et al. is used. ${ }^{8)}$ To simulate the transonic flow on an airfoil with an aileron in forced oscillations, the two-dimensional Navier-Stokes equations are analyzed. Thin-layer approximation is adopted for the boundary layer using the Baldwin-Lomax turbulence model ${ }^{9)}$ under the assumption that there are no large-scale boundary layer separations. The Yee-Harten Total Variation Diminishing (TVD) method ${ }^{10)}$ is used to discretize the governing equations and the Alternating Direction Implicit (ADI) method to integrate them. After acquiring a steady-flow field, the unsteady flow is processed in a time accurate manner with an appropriate time step using the steady-flow field as the initial condition.

In order to validate the present CFD code, comparisons between the simulated and experimental ${ }^{11)}$ results are shown in Figs. 1 and 2. For the comparisons, the NACA64A006 airfoil is used. The flow conditions are summarized in Table 1. The grid used here is the $\mathrm{C}$ type, which is 209 by 80. Figure 1 shows the comparison of the flows without shock waves. Between the simulated and the experimental results, the steady and unsteady pressure distributions show good agreement, while there are discrepancies in the peak values of the steady pressures and the real component of the unsteady pressures.

Figure 2 shows a comparison between the cases including a shock wave. In order to compensate the differences of the steady pressure distributions between the simulation and the experiment, the condition applied to the simulation is slightly different from the experimental one. In the unsteady results, the peak values shown in the simulation are smaller than those in the experiments, the same as Fig. 1. But the aspect of the distributions does not differ largely from the experiment.

From the above comparisons, it is validated that the characteristics of the flows around the airfoil can be appropriately simulated for both cases, with and without shock waves.

Table 1. Calculated conditions for CFD code verification (NACA64A006).

\begin{tabular}{|c|c|c|}
\hline & \multicolumn{2}{|c|}{ Fig. 1} \\
\hline & Experiment & Simulation \\
\hline$M$ & 0.804 & 0.804 \\
\hline$R e$ & $2.35 \times 10^{6}$ & $2.35 \times 10^{6}$ \\
\hline$\alpha[\operatorname{deg}]$ & 0.0 & 0.0 \\
\hline$\delta_{\mathrm{o}}[\mathrm{deg}]$ & 0.0 & 0.0 \\
\hline \multirow[t]{3}{*}{$k$} & 0.253 & 0.253 \\
\hline & \multicolumn{2}{|c|}{ Fig. 2} \\
\hline & Experiment & Simulation \\
\hline$M$ & 0.879 & 0.860 \\
\hline $\operatorname{Re}$ & $2.44 \times 10^{6}$ & $2.44 \times 10^{6}$ \\
\hline$\alpha[\mathrm{deg}]$ & 0.0 & 0.9 \\
\hline$\delta_{\mathrm{o}}[\mathrm{deg}]$ & 0.0 & 0.0 \\
\hline$k$ & 0.234 & 0.234 \\
\hline
\end{tabular}




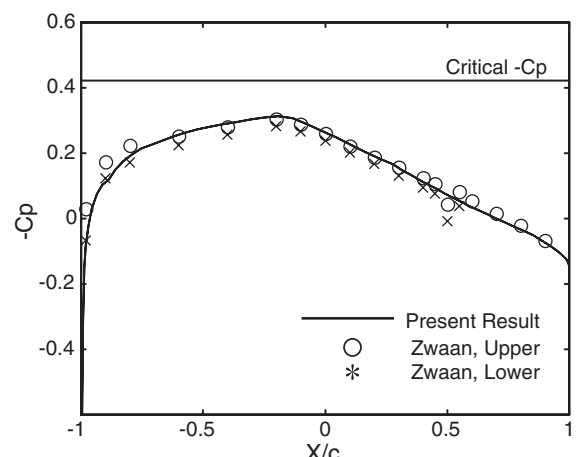

(a) Steady

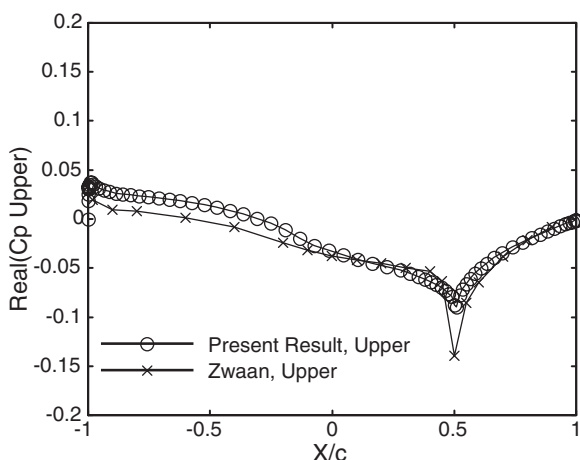

(c) Unsteady / Real

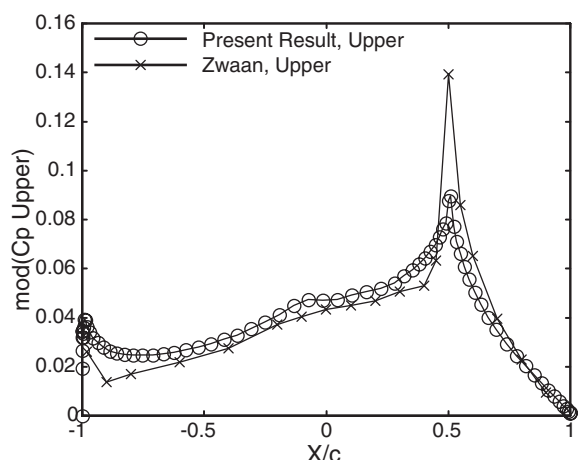

(b) Unsteady / Amplitude

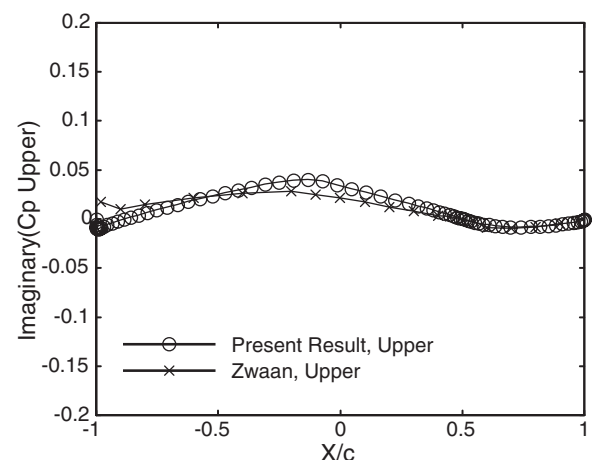

(d) Unsteady / Imaginary

Fig. 1. Comparison of pressure distributions without shock waves. (NACA64A006, $M=0.804, R e=2.35 \times 10^{6}, k=0.253$ )

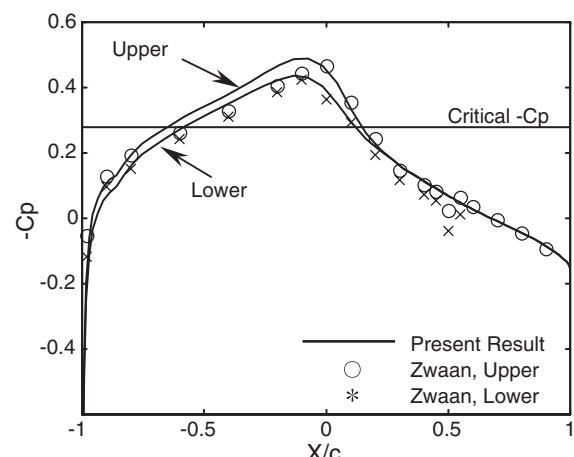

(a) Steady

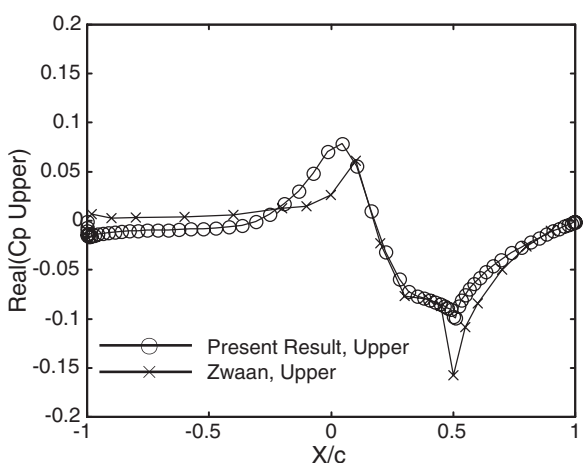

(c) Unsteady / Real

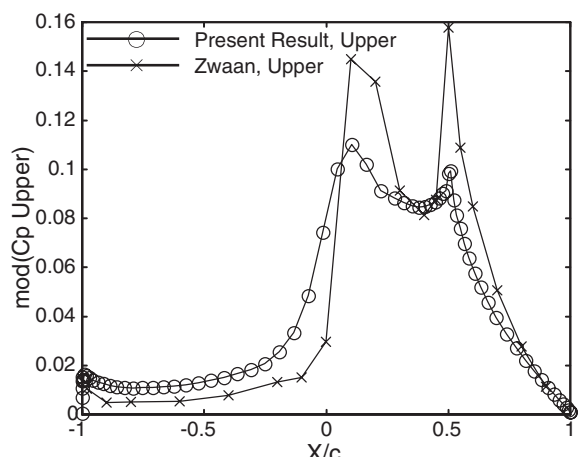

(b) Unsteady / Amplitude

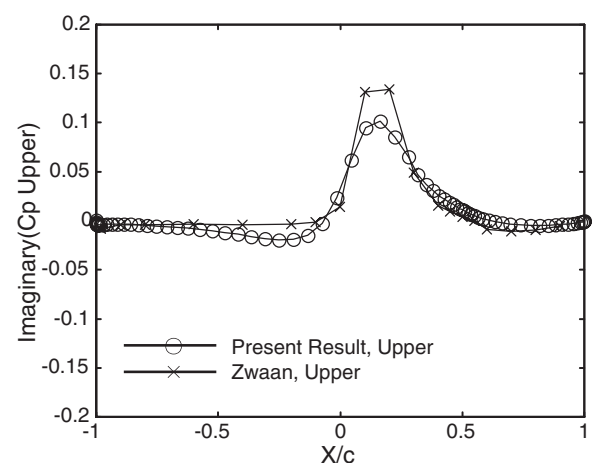

(d) Unsteady / Imaginary

Fig. 2. Comparison of pressure distributions with a shock wave. (NACA64A006, $M=0.879, R e=2.44 \times 10^{6}, k=0.234$ ) 
Table 2. Calculated conditions for simulations (NACA0003).

\begin{tabular}{|c|c|c|}
\hline & \multicolumn{2}{|c|}{ Aileron length $0.094 c$} \\
\hline & $\delta_{\mathrm{o}}=0 \mathrm{deg}$ & $\delta_{\mathrm{o}}=5 \mathrm{deg}$ \\
\hline \multirow[t]{3}{*}{$M$} & $0.700,0.750,0.800$ & $0.700,0.750,0.800$, \\
\hline & $0.850,0.900,0.930$ & $0.850,0.875,0.900$ \\
\hline & $0.940,0.945,0.950$ & $0.910,0.930,0.940$ \\
\hline $\operatorname{Re}$ & $1.1 \times 10^{7}$ & $1.1 \times 10^{7}$ \\
\hline$\alpha[\mathrm{deg}]$ & 0.0 & 0.0 \\
\hline$\delta_{\text {amp }}[\mathrm{deg}]$ & 2.0 & 2.0 \\
\hline \multirow[t]{3}{*}{$k$} & 0.325 & 0.325 \\
\hline & \multicolumn{2}{|c|}{ Aileron length $0.25 c$} \\
\hline & $\delta_{\mathrm{o}}=0 \mathrm{deg}$ & $\delta_{\mathrm{o}}=5 \mathrm{deg}$ \\
\hline \multirow[t]{2}{*}{$M$} & $0.800,0.920,0.935$ & $0.800,0.840,0.850$ \\
\hline & $0.942,0.950$ & $0.870,0.900,0.950$ \\
\hline $\operatorname{Re}$ & $1.0 \times 10^{7}$ & $1.0 \times 10^{7}$ \\
\hline$\alpha[\mathrm{deg}]$ & 0.0 & 0.0 \\
\hline$\delta_{\text {amp }}[\operatorname{deg}]$ & 2.0 & 2.0 \\
\hline$k$ & 0.2 & 0.2 \\
\hline
\end{tabular}

\section{Conditions of Simulations}

Table 2 shows the conditions simulated in the present studies. The airfoil used here is NACA0003 with an aileron. Two different aileron lengths, $0.25 c$ and $0.094 c$, are considered in order to study the effects of the aileron length. The former case is often studied to describe unsteady aerodynamic characteristics around an aileron. ${ }^{11,12)}$ The latter is the same configuration as the experimental model, ${ }^{3-5)}$ whose test results are presented in paragraph 4.5. In order to change the shock wave location on the airfoil, the Mach number is appropriately selected. In the simulations for the shorter aileron, the Reynolds number, $\operatorname{Re}(=\rho U c / \mu)$, and the reduced frequency, $k(=\pi f c / U)$, are set to almost the same values as the experimental condition. On the other hand, for the cases of the longer aileron, $R e$ and $k$ are defined in a different manner from the shorter one, although the difference is slight. The results acquired for both aileron lengths, therefore, can be compared without loss of generality. The effect of the mean deflection angle of aileron, $\delta_{0}$, on the flow field and the aileron hinge moment is also considered. The aileron is in a sinusoidal oscillation with an amplitude of $2 \mathrm{deg}$. Figure 3 shows the grid used in the present calculations [C type, $214 \times 80]$.

\section{Results and Discussions}

\subsection{Steady pressure distributions}

At first, the manner to identify the shock wave location is described. Figure 4 shows the steady pressure distribution on the NACA64A006 airfoil. The actual shock wave location is acquired using visualization technology by Tijdeman, ${ }^{12)}$ in which the shock wave is located at $x / c=$ 0.582 . On the other hand, the shock wave location is generally identified from the pressure distributions as the point where the pressure gradient has the maximum value. The

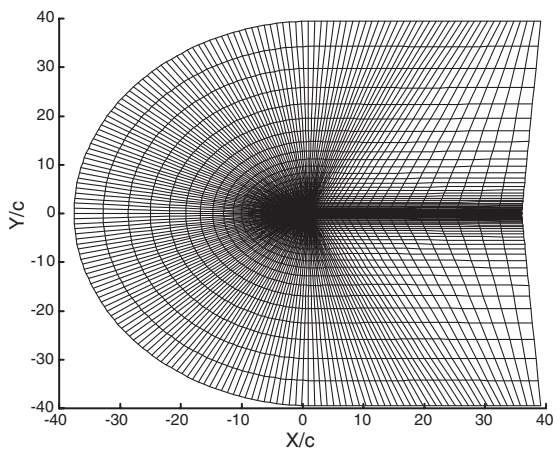

(a) Whole View

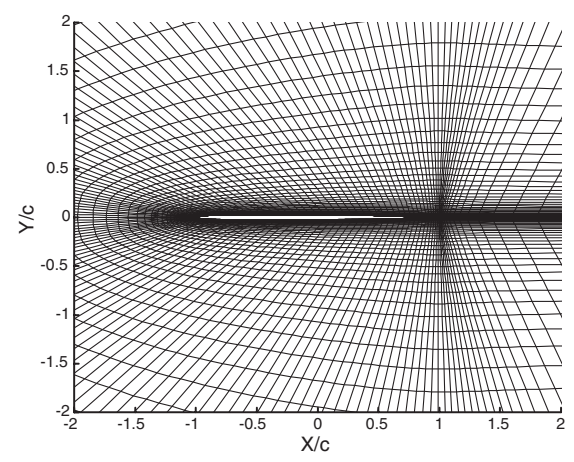

(b) Near the Wing

Fig. 3. Computational grid. (C type, $214 \times 80$, NACA0003)

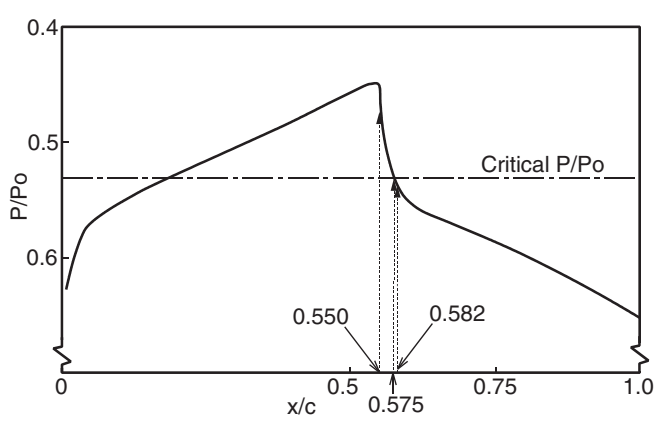

Fig. 4. Detection of shock wave location. (Pressure distribution: Ref. 12), NACA64A006)

maximum pressure gradient locates at $x / c=0.550$ in this case. As shown in Fig. 4, there is a large difference between these two locations. In order to acquire a better estimation of the shock wave location from the pressure distributions, it is simply defined as the point where the pressure value agrees with the critical one in the present study. This results in the shock wave location at $x / c=0.575$, which is much closer to the actual value than $x / c=0.550$.

Figures 5(a) and 5(b) show the steady pressure distributions for the cases of a $0.25 c$ aileron length. The steady pressure is translated to the following pressure coefficient.

$$
C_{p}=\frac{P-P_{s}}{q}
$$

Figure 5(a) is for cases of $\delta_{0}=0 \mathrm{deg}$. At the Mach number of $0.800,-C_{p}$ is lower than the critical one everywhere on the airfoil. At higher Mach numbers at which shock waves 


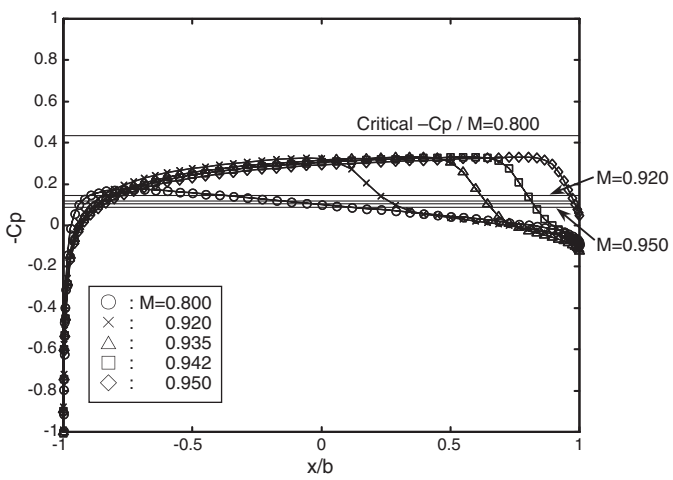

(a) $\delta \mathrm{o}=0 \mathrm{deg}$

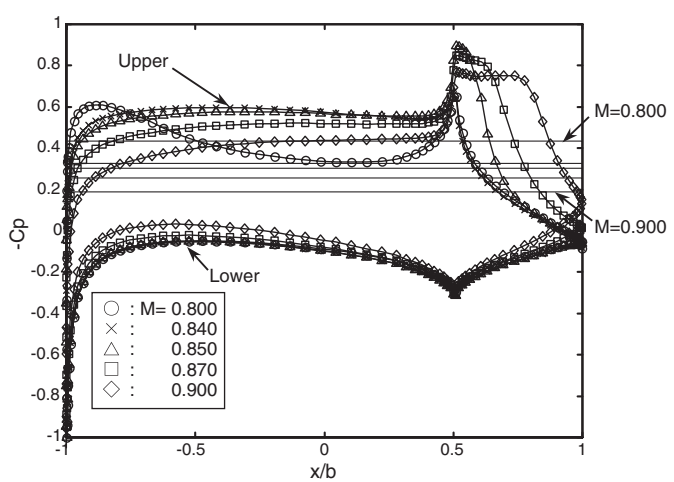

(b) $\delta o=5 \mathrm{deg}$

Fig. 5. Steady pressure distributions.

(NACA0003, Aileron length $=0.25 c$ )

appear on the airfoil, the steady shock wave moves downstream on the airfoil as the Mach number increases. The $-C_{p}$ distributions downstream of the shock wave do not increase excessively, and therefore, trailing edge separations are not expected.

Figure 5(b) is for $\delta_{\mathrm{o}}=5 \mathrm{deg}$. There exists a pressure peak at the aileron's leading edge. On the upper surface, the flow accelerates at the aileron's leading edge. As the Mach number increases, the shock wave location moves downstream on the aileron.

\subsection{Unsteady pressure distributions}

Figures 6(a) and 6(b) show unsteady pressure distributions for the cases of a $0.25 c$ aileron length. In these figures, the unsteady pressure is expressed as the following pressure coefficient, $C_{p_{-} \text {unst }}$.

$$
C_{p_{\text {_unst }}}=\frac{P_{\mathrm{unst}}-P_{s}}{q}
$$

$P_{\text {unst }}$ has a phase lag from the aileron oscillation. $P_{s}$ and $q$ are steady values. $C_{p \_ \text {unst }}$ has the same phase lag as $P_{\text {unst }}$, and it is decomposed into real and imaginary components by the following relation. Here, $C_{p_{-} \text {unst }}$ is expressed as the value normalized with the aileron motion phase. The aileron deflection angle, $\delta$, is also shown.

$$
\begin{aligned}
C_{p \_ \text {unst }} & =C_{p \_ \text {amp }} \cdot e^{-i \theta} \\
& =C_{p \_ \text {amp }} \cos \theta-i C_{p \_ \text {unst }} \sin \theta \\
& =C_{p}^{\prime}+i C_{p}^{\prime \prime}
\end{aligned}
$$

$$
\delta=\delta_{\mathrm{o}}+\delta_{\mathrm{amp}} \cdot e^{i 2 \pi f t}
$$

In Fig. $6, C_{p \_ \text {amp }}, C_{p}^{\prime}$ and $C_{p}^{\prime \prime}$ are presented against the chordwise position, $x / c$. The simulated results only on the upper surface are presented here.

Figure 6(a) shows the cases of $\delta_{\mathrm{o}}=0 \mathrm{deg}$. As described in the previous section, the steady shock wave location on the airfoil moves rearward as the Mach number increases. In the amplitude distributions, when a shock wave locates downstream far from the aileron hinge, a flat distribution appears on the aileron. In the distributions of the imaginary component, the peak appears near the mean shock wave location. For the case of $M=0.920$, this peak is blunt and wide as compared to those for other Mach number cases. This distribution is caused by the broad shock wave motion.

Figure 6(b) shows the cases of $\delta_{\mathrm{o}}=5 \mathrm{deg}$. On the aileron, the amplitude distribution changes at $M=0.870$ when the free-stream Mach number increases. That is, while the peak value decreases at the aileron hinge, the amplitude increases rearward of the aileron. This characteristic also appears in the distributions of the real component, though the peak value shows a minus sign. In the distributions of the imaginary component, the peak shows the maximum value at $M=$ 0.840 , but it does not strongly influence the imaginary component of the aileron hinge moment, which is presented later in this paper.

\subsection{Shock wave motions}

Figures 7(a) and 7(b) show the time-stepped shock wave motion for the cases of $0.25 c$ aileron length. The position, $x / b=0.5$, corresponds to the aileron's leading edge. Smooth curves are obtained by linearly interpolating the discrete results from the simulation. The solid lines with symbols indicate the shock wave motion at the Mach numbers corresponding to the legends. The solid line alone shows the phase of the aileron motion, which is sinusoidal.

Figure 7(a) is for $\delta_{0}=0 \mathrm{deg}$. The mean location of the shock wave motion moves rearward as the Mach number increases. The curve is not continuous for $M=0.920$. This is caused by the drawing way. The shock wave motions are presented in Fig. 7 in such a way that the location of only the most rearward shock wave is represented. For the case of $M=0.920$, two shock waves appear on the airfoil, one in front of the aileron's leading edge and one on the aileron surface. The latter one can exist during only part of a cycle. Therefore, at the time when the shock wave appears on the aileron, abrupt shock wave movement appears in Fig. 7(a). At $M=0.942$, shock wave motion is limited to the region on the aileron. At $M=0.950$, the shock wave almost reaches the aileron's trailing edge.

Figure 7(b) is for $\delta_{\mathrm{o}}=5 \mathrm{deg}$. The shock wave appears and reaches the aileron's trailing edge at lower Mach numbers than those of $\delta_{\mathrm{o}}=0 \mathrm{deg}$. There exist discontinuities in the shock wave locations at $M=0.800$ and 0.840 . These are caused by the same reason as that explained in Fig. 7(a).

Figures 7(a) and 7(b) are the shock wave motions on the upper surface of the airfoil. For the case of $\delta_{\mathrm{o}}=0 \mathrm{deg}$, the shock wave motions on the lower surface are exactly out- 


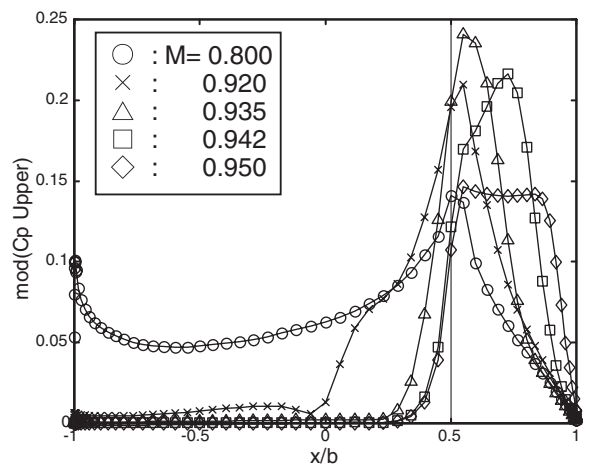

(i) Amplitude

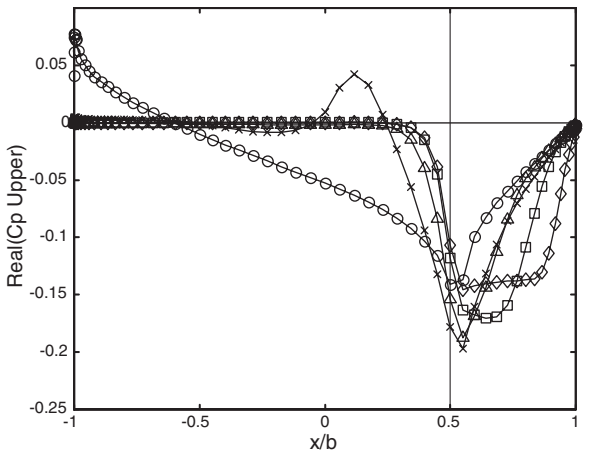

(ii) Real Component

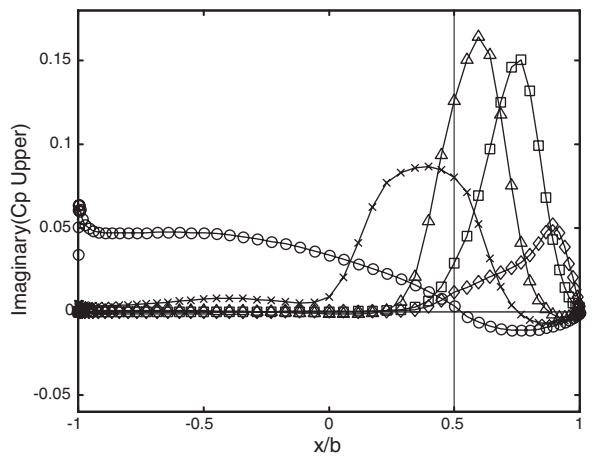

(iii) Imaginary Component

(a) $\delta o=0 \mathrm{deg}$

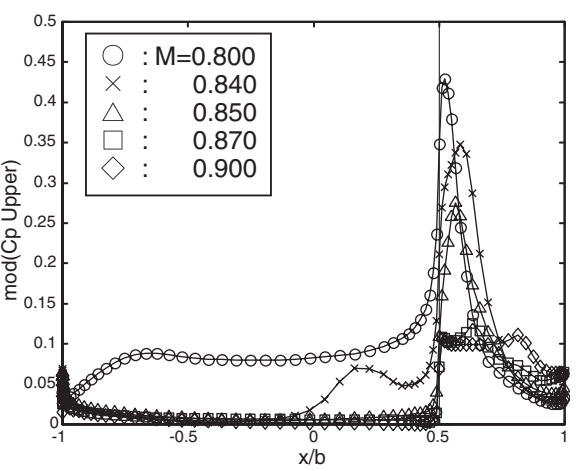

(i) Amplitude

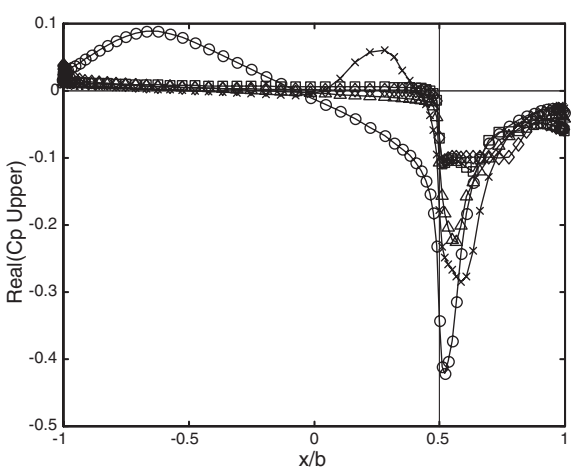

(ii) Real Component

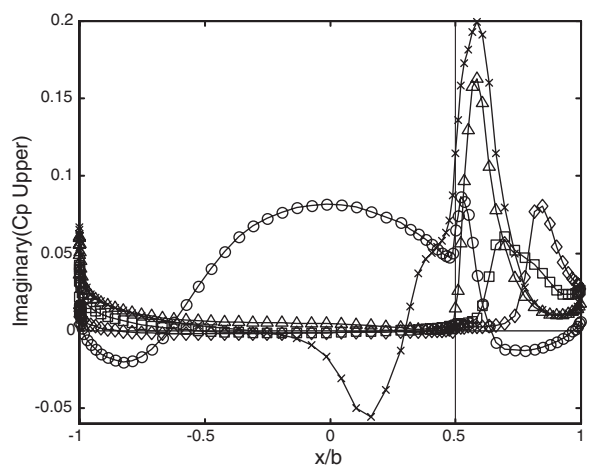

(iii) Imaginary Component

(b) $\delta o=5 \mathrm{deg}$

Fig. 6. Unsteady pressure distributions

(NACA0003, Aileron length $=0.25 c$ )

of-phase with those on the upper surface. For the case of $\delta_{\mathrm{o}}=5 \mathrm{deg}$, the shock wave appears on the lower surface at a higher Mach number than it does on the upper surface, and its motion does not strongly influence the aileron hinge moment (AHM). The shock wave motions only on the upper surface are, therefore, presented in this paper.

Figure 8 shows the relationship between the mean location and the oscillating width of the shock wave motions on the aileron's upper surface. The cases of different aileron lengths are shown together in Fig. 8 with respect to the mean location and the oscillating width normalized with the aileron length. On the process to calculate the width of the shock wave oscillation, the region only on the aileron is used. That is, if a shock wave oscillates upstream and downstream of the aileron leading edge, only the shock wave mo- tion on the aileron surface is taken into account.

It is found that the shock wave motions are expressed with a universal curve for each $\delta_{0}$ case. The shock wave oscillates in a wider range for $\delta_{\mathrm{o}}=0 \mathrm{deg}$ than for $\delta_{\mathrm{o}}=5 \mathrm{deg}$. For the case of $\delta_{0}=0 \mathrm{deg}$, the shock wave moves in the widest range, where it is around the aileron's mid-chord.

\subsection{Aileron hinge moment}

The unsteady AHM is calculated using the following expression.

$C_{M \text { hinge_unst }}=\frac{1}{\left(c-x_{\mathrm{h}}\right)^{2}} \int_{x_{\mathrm{h}}}^{c}\left(x-x_{\mathrm{h}}\right) \cdot\left(C_{p \_ \text {unst_U }}-C_{p \_ \text {unst_L }}\right) \mathrm{d} x$

The reference length is the aileron length. The hinge moment forcing the aileron's trailing edge downward is defined 


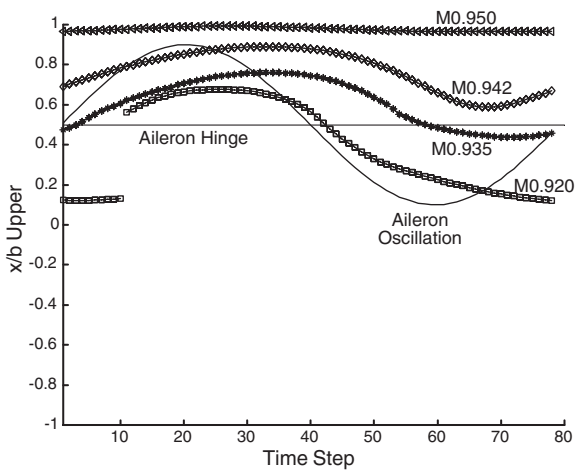

(a) $\delta o=0$ deg. (Aileron Length: $0.025 \mathrm{c}$ )

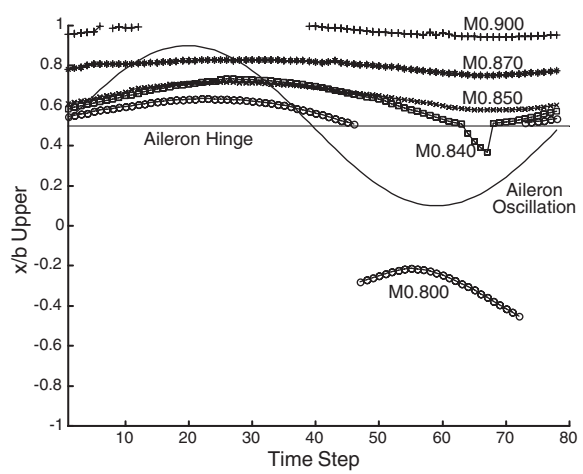

(b) $\delta o=5$ deg. (Aileron Length: $0.025 \mathrm{c}$ )

Fig. 7. Time-stepped shock wave motion. (Aileron length $=0.25 \mathrm{c}$ )

as positive. In order to consider the stability of the aileron, the phase lag of the first harmonic component of AHM from the aileron motion is of importance. The reason is that it dominates if the energy comes into or goes out of the aileron from/to the surrounding flow. Therefore, from the viewpoint of stability, it is sufficient to take AHMI into account in this section.

Figure 9 shows AHMI against the Mach number. As compared with Fig. 7, the values of AHMI become positive when the shock wave starts to oscillate mainly on the aileron

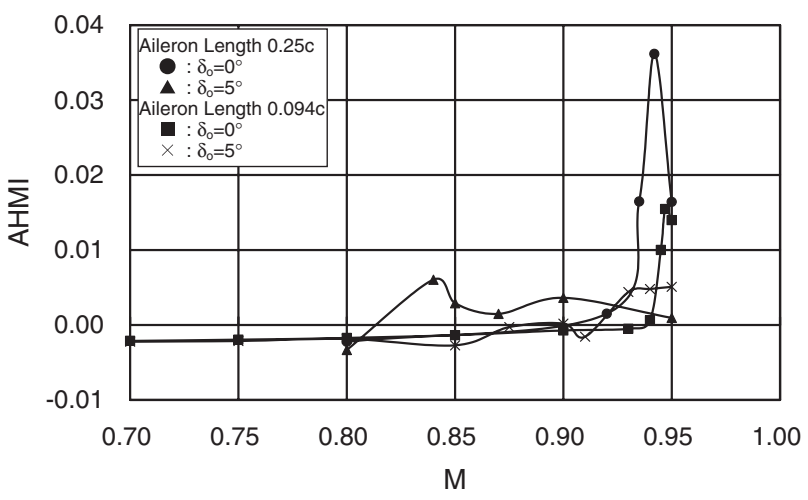

Fig. 9. Imaginary component distributions of aileron hinge moment.

surface. AHMI shows a greater value for the cases of $\delta_{\mathrm{o}}=$ 0 deg than those for the cases of $\delta_{\mathrm{o}}=5 \mathrm{deg}$. The peaks appear for the cases of $\delta_{\mathrm{o}}=0 \mathrm{deg}$ when the shock wave oscillates around the aileron's mid-chord. To see the shock wave motions corresponding to these peaks, the marks $\square$ and $\bigcirc$ are put at those cases in Fig. 8. For $\delta_{\mathrm{o}}=0 \mathrm{deg}$, the effect of the aileron length appears clearly in Fig. 9. AHMI on the longer aileron shows greater value than that on the shorter one. While the shock wave motion is expressed with a unique curve in Fig. 8, the AHMI distributions cannot be done. It is simply imaged that, if the shock wave oscillates wider, the airfoil surface pressure fluctuates with greater amplitude inside the shock wave oscillation region. Therefore, the amplitude of the pressure fluctuations is decided not by the normalized width of the shock wave motion, but by the dimensional width.

\subsection{Comparisons between the results obtained by sim- ulation and experiment}

Concerning unsteady aerodynamics on the SST arrow wing with a partial inboard aileron, experimental data are available in other literature 3-5). The planform of the wing is shown in Fig. 10. The wing has the NACA0003 airfoil at every semi-span section. In the inboard, there is an aileron

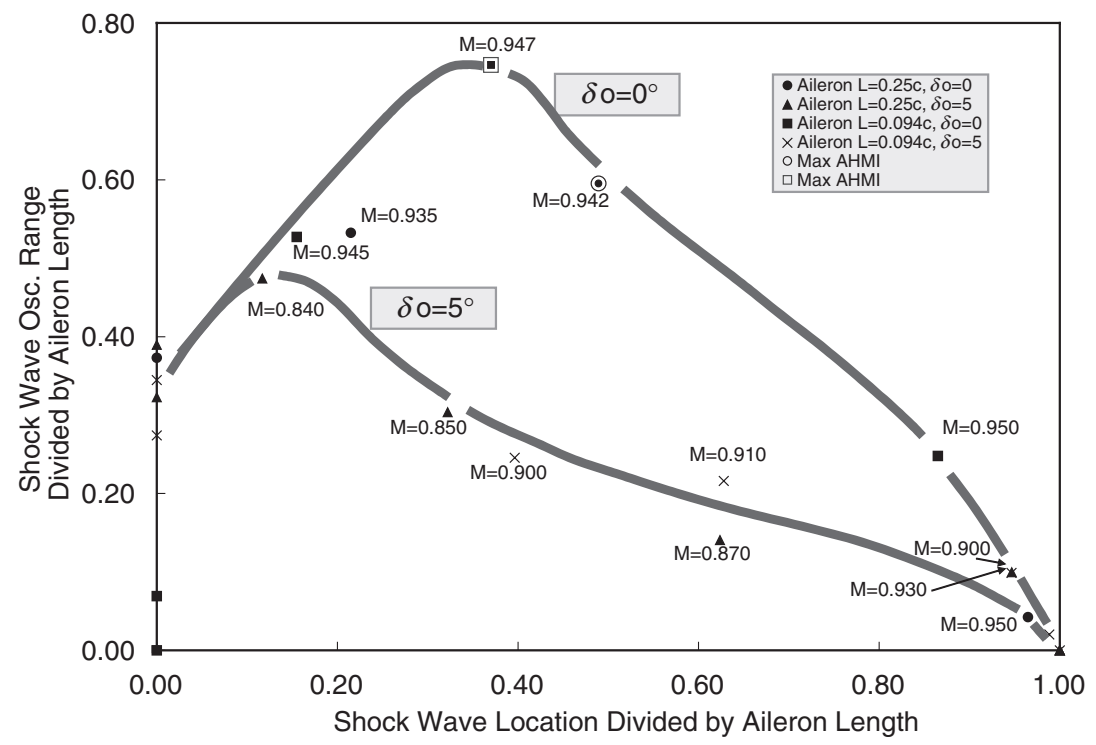

Fig. 8. Relationship between shock wave mean location and oscillating width. 


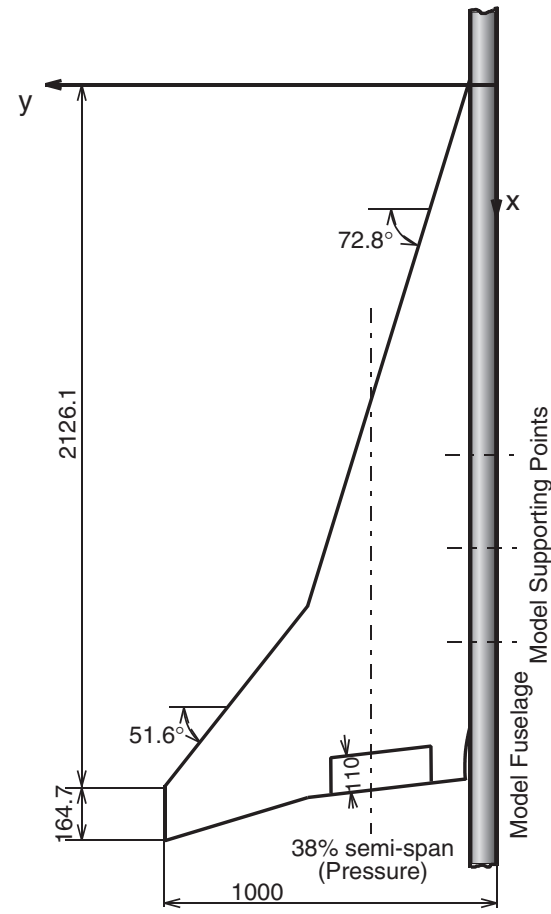

Fig. 10. Plan figure for experimental model.

Table 3. Conditions for comparison (NACA0003).

\begin{tabular}{cccc}
\hline \multicolumn{2}{c}{ Experiment } & Simulation \\
\hline$M$ & 0.9508 & 0.9001 & $0.700,0.750,0.800$, \\
& \multicolumn{3}{c}{$0.850,0.875,0.900}$, \\
& & $0.910,0.930,0.940$, \\
& & 0.950 \\
$R e$ & $1.98 \times 10^{7 *}$ & $1.97 \times 10^{7 *}$ & $1.10 \times 10^{7}$ \\
$\alpha[\mathrm{deg}]$ & 0.0 & 0.0 & 0.0 \\
$\delta_{\mathrm{o}}[\mathrm{deg}]$ & 5.0 & 5.0 & 5.0 \\
$k$ & 0.322 & 0.339 & 0.325 \\
$\delta_{\mathrm{amp}}[\mathrm{deg}]$ & 1.01 & 0.96 & 2.00 \\
\hline
\end{tabular}

* $R e$ is based on the root chord.

that can be oscillated with an electric motor. Unsteady pressure sensors are installed at the $38 \%$ semi-span position, whose section aileron length is $0.094 c$. In this section, the simulated results are compared with the experimental ones. The conditions for the simulations and the experiments are presented in Table 3. For the experimental results, the cases of $\delta_{\mathrm{o}}=5 \mathrm{deg}$ are in focus, because continuous shock waves appear only in these cases.

In this section, we particularly discuss the flow on the aileron. The difference of the experimented 3D flows from the simulated 2D flows is the existence of a side flow on the wing. The flow on the experimental model's aileron, therefore, has a certain flow angle against the aileron's leading edge, which is parallel to the aileron hinge axis. In this circumstance, the flow component perpendicular to the aileron hinge axis influences the aerodynamic forces acting on the aileron surface. The Mach number of the flow component perpendicular to the aileron hinge axis, $M n 2$, which is schematically shown in Fig. 11, can be stated by the following expression.

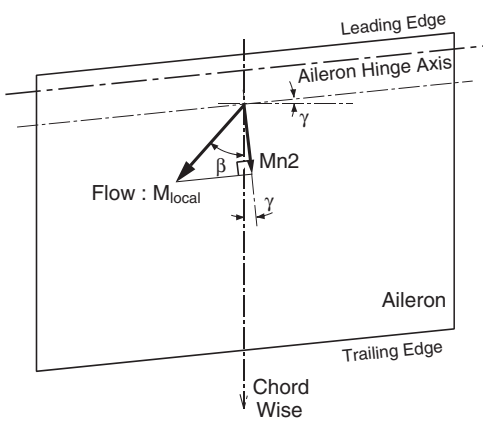

Fig. 11. Explanation of $M n 2$.

$$
\operatorname{Mn} 2=M_{\text {local }} \cdot \cos (\beta+\gamma)
$$

Here, $M_{\text {local }}$ is the Mach number of the local flow just downstream of the aileron's leading edge; $\beta$ is the angle of the local flow from the chord-wise axis; and $\gamma$ is the angle of the aileron's leading edge from the span-wise axis. In the following discussion, $M n 2$ is taken as the parameter to compare the experimental and the simulated results.

Figure 12 shows the shock wave oscillations against $M n 2$. The solid lines accompanied with filled circles and diamond marks show the extent of the shock wave motions. As stated above, for the experiments, shock waves oscillate continuously only at $\delta_{\mathrm{o}}=5 \mathrm{deg}$ Therefore, only the results simulated at $\delta_{\mathrm{o}}=5 \mathrm{deg}$ are presented in this paragraph. In the experimental results, the practical aileron deflection is considered less than $5 \mathrm{deg}$. This is because the flow approaching the aileron is subsonic, it accelerates at the aileron's leading edge, and then becomes supersonic. The calculated possible and maximum flow deflection angle at the aileron's leading edge are 3.7 and $4.2 \mathrm{deg}$ for $M=0.9001$ and $M=0.9508$, respectively. For the experimental result acquired at $M=$ 0.9508 , because of an insufficient number of pressure sensors in the aileron's trailing edge area, the shock wave motion does not form a continuous curve, but shows an intermittent curve. For this case, continuous shock wave oscillation is expected from the existing intermittent curve. Therefore, in Fig. 12, the range of shock wave oscillation is extended to the appropriate length with a dotted line. The aileron oscillation amplitudes measured in the experiments are $1.01 \mathrm{deg}$ and $0.96 \mathrm{deg}$ for $M=0.9001$ and $M=$

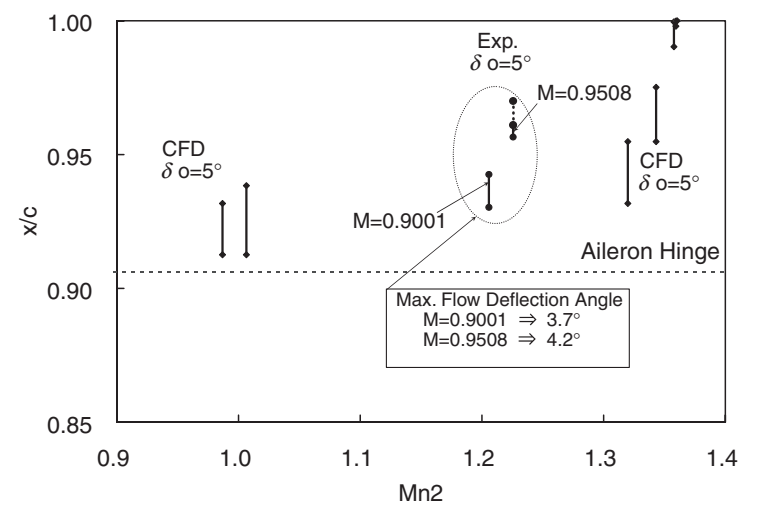

Fig. 12. Comparison of shock wave motion between simulated and experimental results. 


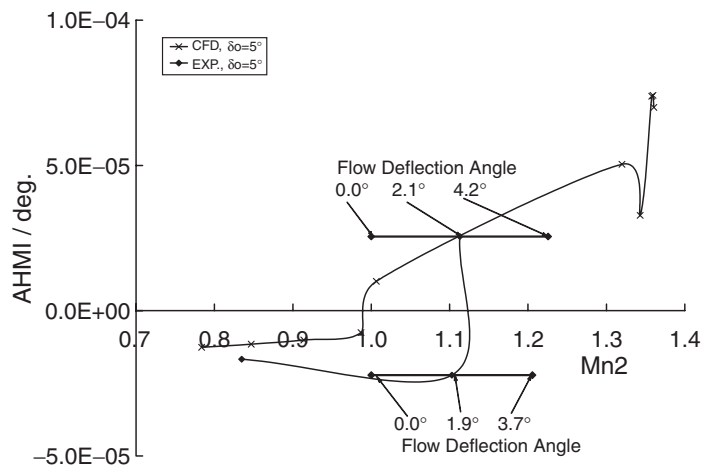

Fig. 13. Imaginary component distributions of aileron hinge moment/ comparison between simulated and experimental results.

0.9508 , respectively, which are smaller values than the simulated amplitude, $2 \mathrm{deg}$. Therefore, in the experiments, the shock waves oscillate in smaller regions than those shown in the simulations.

Figure 13 shows the AHMI distributions against $M n 2$, which are normalized with the aileron oscillation amplitude. For convenience, the curve is drawn through the center of the $M n 2$ range for the experimental results. The simulated result becomes positive when $M n 2$ is beyond 1.0. For the experimental case $M=0.9001$, AHMI shows a negative value. Furthermore, from Fig. 12, the shock wave oscillation region lies on the front area of the aileron, which is, in the simulated results, the area that $M n 2$ is close to 1.0. Therefore, the experimental case $M=0.9001$ might have a $M n 2$ value close to 1.0 , which means that the flow deflection passing the aileron's leading edge is close to $0.0 \mathrm{deg}$. The tendency of AHMI changing against Mn2 shows the same characteristics for both the simulated and the experimental results. That is, as the shock wave oscillates more rearward on the aileron, AHMI increases and reaches a positive value.

\section{Conclusion}

Two-dimensional CFD simulations were conducted to study transonic flows around the NACA0003 airfoil with an aileron. The aileron is in a forced sinusoidal oscillation. Navier-Stokes equations were analyzed for the simulation. Two different aileron lengths were considered. The Mach number was varied to change the steady shock wave location. The present study is summarized as follows.

1. The imaginary component of the unsteady pressure has a peak near the mean location of the shock wave motion.

2. The shock wave location is defined as the point where the local pressure is equal to the critical pressure. The validation of this definition has been made through the experimental pressure data.

3. The shock wave motions are expressed with a universal curve for each of different $\delta_{0}$ : here, $\delta_{\mathrm{o}}$ is the mean de- flection angle of the aileron. This conclusion can be true if the shock wave motions are normalized with the aileron length.

4. The amplitude of shock wave motion on the aileron becomes smaller as $\delta_{\mathrm{o}}$ increases from $0.0 \mathrm{deg}$ to $5.0 \mathrm{deg}$.

5. For $\delta_{\mathrm{o}}=0 \mathrm{deg}$, the shock wave oscillates in the widest range around the aileron's mid-chord. At this condition, the imaginary component of aileron hinge moment (AHMI) is positive and at its greatest value.

6. The AHMI value varies when the aileron length changes even if the non-dimensional shock wave motion does not change, because AHMI is strongly influenced by the dimensional amplitude of the shock wave motion.

7. The AHMI distributions against Mn2 show common characteristics between the simulated and the experimental results. As the shock wave oscillates more rearward on the aileron, the AHMI value increases and reaches a positive value.

\section{References}

1) Kheirandish, H. R. and Nakamichi, J.: Simulation of a Flexible Aircraft Dynamics, Proceedings of JSASS 13th International Sessions in 37th Aircraft Symposium, 1999, pp. 665-668.

2) Lambourne, N. C.: Control-Surface Buzz, Aeronautical Research Council R\&M No. 3364, 1964.

3) Tamayama, M., Saitoh, K., Matsushita, H., Nakamichi, J. and Andoh, Y.: Experimental Analysis of Unsteady Aerodynamics around an SST Elastic Wing Model, Proceedings of CEAS/AIAA/ICASE/NASA LaRC International Forum on Aeroelasticity and Structural Dynamics, 1999.

4) Tamayama, M., Saitoh, K., Matsushita, H., Hashidate, M. and Nakamichi, J.: Measurements of Unsteady Pressure Distributions and Dynamic Deformations on an SST Elastic Arrow-Wing Model, Trans. Jpn. Soc. Aeronaut. Space Sci., 42 (1999), pp. 92-100.

5) Tamayama, M., Saitoh, K., Matsushita, H. and Nakamichi, J.: NAL SST Arrow Wing with Oscillating Flap, RTO (Research \& Technology Organization, NATO) TR-26, Chapter 12 of Verification and Validation Data for Computational Unsteady Aerodynamics, 2000, pp. 295-318.

6) Bendiksen, O. O.: Nonclassical Aileron Buzz in Transonic Flow, AIAA Paper 93-1479, 1993.

7) Tamayama, M.: A Study on Unsteady Transonic Flows around a Thin Wing with a Flap, a thesis of Ph.D. degree of Science University of Tokyo, 2001 (in Japanese).

8) Kheirandish, H. R., Beppu, G. and Nakamichi, J.: Numerical Solutions of Inviscid \& Viscous Flows about Airfoils by TVD Method, NAL SP27, 1994, pp. 135-140.

9) Baldwin, B. S. and Lomax, H.: Thin Layer Approximation and Algebraic Model for Separated Turbulent Flows, AIAA Paper 78-257, 1978.

10) Yee, H. C. and Harten, A.: Implicit TVD Schemes for Hyperbolic Conservation Laws in Curvilinear Coordinates, AIAA J., 25 (1987), pp. 266-274.

11) Zwaan, R. J.: NACA64A006. Oscillating Flap, Compendium of Unsteady Aerodynamic Measurements, AGARD-R-702, 1982.

12) Tijdeman, H.: Investigations of the Transonic Flow around Oscillating Airfoils, NLR TR 77090 U, 1977. 